\title{
Precise GRB source locations from the renewed interplanetary network
}

\author{
T.L. Cline ${ }^{1}$, S. Barthelmy ${ }^{1}$, P. Butterworth ${ }^{1}$, F. Marshall ${ }^{1}$, T. McClanahan ${ }^{1}$, D. Palmer ${ }^{1}$, J. Trombka $^{1}$, K. Hurley $^{2}$, \\ R. Gold ${ }^{3}$, R. Aptekar ${ }^{4}$, D. Frederiks ${ }^{4}$, S. Golenetskii ${ }^{4}$, V. Il'Inskii ${ }^{4}$, E. Mazets ${ }^{4}$, G. Fishman ${ }^{5}$, C. Kouveliotou ${ }^{5}$, and \\ C. Meegan $^{5}$ \\ 1 NASA-GSFC, Greenbelt, Maryland 20771, U.S.A. \\ e-mail: cline@apache.gsfc.nasa.gov \\ 2 UCB-SSL, Berkeley, CA 94720, U.S.A. \\ 3 APL, Laurel, MD 20723, U.S.A. \\ ${ }^{4}$ Ioffe PTI, St. Petersburg, 194021 Russia, U.S.A. \\ 5 NASA-MSFC, Huntsville, AL 35812, U.S.A.
}

Received December 29, 1998; accepted February 24, 1999

\begin{abstract}
A new interplanetary network (IPN) is made possible by the modification of the NEAR mission, now at 2 AU distance. With Ulysses and the near-Earth GRB monitors, this fully long-baseline network will produce GRB localizations at a rate of about one per week, with accuracies varying from several to tens of arc minutes. Given the delays inherent in the collection of deep space data, high-precision alerts with delays under one day should be possible at a rate of one to several per month. An automatic analysis system is being developed to optimize the process.
\end{abstract}

Key words: gamma-rays: bursts — instrumentation: miscellaneous

\section{History}

The BeppoSAX mission has provided the missing link connecting gamma ray bursts to their cosmological sources. The discovery of the long-lived soft X-ray component of GRBs assisted in enabling the further discovery of the GRB-associated optical and radio afterglows. Essential to this process was the several-hour rapidity of BeppoSAX's GRB source notification. Long ago in the 1970s, the first IPN had been successfully defining source locations of GRBs to arc-minute accuracies, but the data processing times and the distributions then suffered considerable and unavoidable delays. Following the end of the first IPN in the $1980 \mathrm{~s}$, there were years of IPN catastrophes, such as the cancellation of Solar Polar, the failure of the Mars Observer at Mars, the failure of Mars 96 at launch, and the apparent unavailability of several other missions for GRB monitors or piggybacks.

Send offprint requests to: T.L. Cline

\section{Renewal}

The recent modification of the Near Earth Asteroid Rendezvous (NEAR) for continuous gamma-ray monitoring makes possible the renewal of a burst network with a geometry having three widely separated vertices, required for precise source definition. In an interplanetary trajectory to the asteroid Eros, now over $2 \mathrm{AU}$ from the Earth, NEAR completes the network that includes Ulysses, at up to $6 \mathrm{AU}$, and all the near-Earth burst monitors involved in the GCN (in particular, Konus, on GGS-Wind, at a distance varying up to 5 light-seconds, and the orbiting detectors on Compton-GRO, Rossi-XTE and BeppoSAX). Missions not in Earth orbit generally download data on a daily to several-day basis, but some fraction of the bursts from any preceding day can be, of course, only several hours old. In addition, it is planned that when NEAR uses its low bit-rate mode (when bursts would otherwize be less often recovered), a revision in the spacecraft software will enable prioritized storage and downloading for preselected events. Thus, combined with the GCN, the renewed IPN may provide a number of globally distributed GRB alerts of adequate rapidity and precision to be useful for optical afterglow searches, and an added number with modest delays, adequate for radio astronomical searches.

\section{System}

The new IPN has been self-calibrated with the quite fortuitous occurrence of the giant SGR flare of 1998 August 27 , given its precisely known SGR1900 +14 source location (see IAU Circulars 7002, 7004 and 7005). The Ulysses detector response was not saturated (Hurley et al. 1999), but this 1979-March-5-like event was of sufficient intensity to saturate the larger detectors on GGS-Wind Konus and NEAR. However, the times of a modest precursor and of 
the onset of the largest increase were free of distortion. The resulting analysis gave a source of several arc minutes precisely agreeing with the SGR location. Thus, the IPN detection of future events from known SGRs or of future GRBs localized by BeppoSAX will demonstrate its continued timing stability and calibration.

\section{Capability}

The directional precision of this network is limited by the timing ability of the NEAR monitor. Unlike CGRO BATSE, Ulysses, or the GGS-Wind Konus experiment, this instrument does not have a GRB trigger or profile memory. The modification that was, in fact, heroically undertaken in flight was to enable continuous monitoring of the total gamma-ray rate on a one-second per readout basis. Since event profiles can be compared to somewhat finer resolution than one bin width (only if the event may be complex in profile and of sufficient duration), it is possible that, with NEAR at a distance of over 1200 light-seconds, the precision of the IPN GRB source localization can be as good as \pm 1.5 arcmin.

\section{Process}

The speed of the alert limits the utility of the source fields to be defined by the IPN. The giant SGR event of 1998 August 27 was first reported on the next day by the Konus experiment (CGRO having been below the horizon), using an automatic scheme somewhat analogous to the well-known "BACODINE", now incorporated into the GCN (Barthelmy et al. 1997). A computer-generated search is done through the entire GGS-Wind spacecraft data stream as it is received (before it is split off into separate instrument data, to be sent to the Wind experimenters) to find a gamma-ray burst memory dump. If found, this count-rate history is automatically sent to team members, (there being no source location from the GGS-Wind data alone other than an ecliptic hemisphere preference, unlike the BATSE data distributed by GCN). At present, the IPN team members finish the comparisons themselves, determine the resulting source location, and deliver it to the GCN and the world community. In the case of the prototype giant SGR event in August, that was done within hours of receipt on the next day. This delay time for intervention by humans can and will be eliminated when this process is entirely automated.

\section{Progress}

One step towards that goal, now in place, is the fully automatic comparison of Ulysses data with any GCNdistributed GRB event, to search for an increase in the Ulysses data that may fit the presence of, and light-travel time from, the event seen near the Earth by BATSE or the other participants in the GCN. A successful fit will automatically create a two-spacecraft burst source annulus; this will generally limit the several-degree BATSE source field to a considerably smaller total celestial area. These annular segments are distributed in turn by the GCN to the community. The extension of this process to use NEAR data, as well as Ulysses data, is presently being undertaken, towards the goal of automating GRB source localizations. The other step being pursued is the automation of the NEAR data generation, in analogy to the process in use with Konus data. That is, GRB events will be both first detected in the NEAR data, generating a search in the Ulysses and other spacecraft data files, and vice versa. The last human step, the calculation of the limits of the source field, or "error box", must also be automated before the results can be delivered in the most reliable and rapid manner.

\section{Conclusions}

The new interplanetary GRB network may provide several distinct benefits to the gamma-ray burst community. First, the IPN will be able to promptly define GRB source locations of use to optical astronomers for some months after NEAR enters Eros orbit in 1999, bridging the gap between the present and the launch of HETE-2, expected in early 2000. Second, the IPN will also provide an extended number of GRB source localizations on a somewhat less prompt scale but adequately rapid for radio astronomical work. Third, to possibly complement the remarkable BeppoSAX results that have been generally confined to the longer, complex variety of bursts, the IPN, because of its triggering techniques, should provide results for brief, single-spike events that have been speculated to form a separate population. Finally, it is entirely possible (if the GRB discipline continues to be as unpredictable as before) that some unexpected result could be provided.

\section{References}

Barthelmy S., Butterworth P., Cline T.L., Gehrels N., Marshall F., Takeshima T., Connaughton V., Kippen R.M., Kouveliotou C., Robinson C., 1997, "The GRB Coordinates Network (GCN): A Status Report", AIP 428, Gamma Ray Bursts: Fourth Huntsville Symposium, 99-103

Hurley K., Cline T.L., Mazets E., Barthelmy S., Butterworth P., Marshall F., Palmer D., Aptekar R., Golenetskii S., Il'Inskii V., Frederiks D., McTiernan J., Gold R., Trombka J., 1999, "A Giant, Periodic Flare from the Soft Gamma Repeater SGR1900 + 14", Nat 397, 41-43 